\title{
Could different treatment approaches in acromegaly influence life expectancy? A comparative study between Bulgaria and Campania (Italy)
}

\section{Annamaria Colao, Silvia Vandeva', Rosario Pivonello, Ludovica Francesca Stella Grasso, Emil Nachev', Renata S Auriemma, Krasimir Kalinov ${ }^{2}$ and Sabina Zacharieva'}

Dipartimento di Medicina Clinica e Chirurgia, Sezione di Endocrinologia, Università Federico II di Napoli, via S. Pansini 5, 80131 Naples, Italy, ${ }^{1}$ Clinical Center of Endocrinology and Gerontology 'Akad. Ivan Pentchev' Medical University Sofia, 2 Zdrave Street, 1431 Sofia, Bulgaria and ${ }^{2} \mathrm{New}$ Bulgarian University, 21 Montevideo Street, 1618 Sofia, Bulgaria
Correspondence should be addressed to A Colao

Email

colao@unina.it

\begin{abstract}
Background: Mortality in acromegaly strictly depends on optimal control of GH and IGF1 levels. Modern medical therapy with somatostatin analogs (SSAs) and GH receptor antagonists (GHRAs) is not available in many countries due to funding restrictions. This retrospective, comparative, cohort study investigated the impact of different treatment modalities on disease control (GH and IGF1) and mortality in acromegaly patients.

Methods: Two cohorts of patients with acromegaly from Bulgaria $(n=407)$ and Campania, Italy $(n=220)$, were compared, and mortality rates were evaluated during a 10-year period (1999-2008).

Results: The major difference in treatment approach between cohorts was the higher utilization of SSAs and GHRAs in Italy, leading to a decreased requirement for radiotherapy. Significantly more Italian than Bulgarian patients had achieved disease control (50.1 vs $39.1 \%, P=0.005)$ at the last follow-up. Compared with the general population, the Bulgarian cohort had a decreased life expectancy with a standardized mortality ratio (SMR) of $2.0(95 \% \mathrm{Cl} 1.54-2.47)$ that was restored to normal in patients with disease control - SMR 1.25 (95\% Cl 0.68-1.81). Irradiated patients had a higher cerebrovascular mortality - SMR 7.15 (95\% Cl 2.92-11.37). Internal analysis revealed an independent role of age at diagnosis and last GH value on all-cause mortality and radiotherapy on cerebrovascular mortality. Normal survival rates were observed in the Italian cohort: SMR 0.66 (95\% Cl 0.27-1.36).

Conclusions: Suboptimal biochemical control was associated with a higher mortality in the Bulgarian cohort. Modern treatment options that induce a strict biochemical control and reduce the necessity of radiotherapy might influence the life expectancy. Other factors, possibly management of comorbidities, could contribute to survival rates.
\end{abstract}

European Journal of Endocrinology

(2014) 171, 263-273

\section{Introduction}

Acromegaly is a rare disease with an estimated annual incidence of 3-4 cases/million population and a prevalence of 40-90 cases/million population $(1,2)$. More recent data $(3,4)$ have reported a higher prevalence of one case out of 8000-11000 individuals. Typically, chronic growth hormone (GH) excess is caused by benign pituitary adenoma. Owing to its indolent and insidious nature, diagnosis of acromegaly is often delayed for years (5). Approximately $60 \%$ of deaths are due to cardiovascular disease, $25 \%$ are due to respiratory disease, and $15 \%$ are due to malignancies. When patients have cardiac disease or diabetes mellitus at diagnosis, it is reported that (c) 2014 European Society of Endocrinology Printed in Great Britain
Published by Bioscientifica Ltd. 
mortality occurs within 15 years in almost all patients with cardiac disease and only $20 \%$ of patients with diabetes would survive for 20 years (6). A GH value of $<2.5 \mu \mathrm{g} / \mathrm{l}$ (measured by RIA and probably $<1 \mu \mathrm{g} / 1$ measured by modern sensitive immunoassays) and insulin-like growth factor 1 (IGF1) levels normalized for age constitute the most predictive survival index (7). Thus, optimal control of GH and IGF1 is mandatory to ensure normal life expectancy in patients.

Treatment options currently available include surgery, medical therapy such as dopamine agonists (DAs), somatostatin analogs (SSAs), and GH receptor antagonists (GHRAs), and irradiation (8). While surgery and irradiation are used worldwide, modern medical therapies (i.e. SSAs and GHRAs) are not available in all countries due to their high cost. In Italy, SSAs have been available since 1988 and GHRAs were introduced in 2004. In Bulgaria, due to funding restrictions, none of these drugs were routinely available until 2008. As such, patients with acromegaly in Bulgaria and Italy may show difference in control of acromegaly and mortality as poor biochemical control due to lack of treatment options may lead to an earlier death.

\section{Patients and methods}

\section{Study design}

This was a retrospective, comparative, cohort study. Bulgaria and Campania region in Italy have a similar population of 7364.570 and 5864.662 inhabitants respectively (http://www.nsi.bg/census2011/index.php; http:// www.comuni-italiani.it/15/index.htm). The Departments of Endocrinology in Sofia and Naples are both national referral centers for acromegaly; therefore, the comparison is considered as reliable. However, it should be stated that the Sofia center is the only one dedicated to pituitary diseases in Bulgaria while although the Naples center is well recognized, it is not the only one available, and therefore a greater actual number of more severe cases in the Naples series cannot be ruled out.

\section{Inclusion criteria}

Mortality in both centers was evaluated between January 1st 1999 and December 31st 2008. We included all consecutive patients who were alive by the end of 2008 or deceased during the upper period of time. Acromegaly was diagnosed during the period of 1955-2008. All patients were residents of Bulgaria or the Campania region. The patients followed up in the University Hospital Federico II of Naples from Jan 1st 1997 were included in a registry approved by the Ethical Committee (no. 60/97). Recently, a Bulgarian database including all patients with acromegaly was created and also approved by the local ethical committee (9).

Patients were treated as lost to follow-up in both centers if their last visit has occurred before 2006 (unless deceased). Information regarding these subjects was obtained from local physicians in Campania, whereas in Bulgaria, patients were invited via mail and/or telephone to attend the clinic.

\section{Subjects}

A total of 407 patients treated at the Clinical Center of Endocrinology and Gerontology, Medical University Sofia, Bulgaria, and 220 treated at Naples Federico II University Hospital, Italy, were included in the study. Bulgarian patients diagnosed before 1979 were irradiated as a first line of treatment, whereas subjects diagnosed later were primarily operated. Unsuccessful surgical intervention was a criterion for treatment with DAs. If medical therapy was inefficient, patients were reoperated or irradiated following medical counsel. In the case of irradiation, most of the patients have been treated with DAs until the effect of radiotherapy was set in. Treatment throughout the study was as follows: primary radiotherapy $n=20$ patients, of whom seven were additionally treated with DAs; primary DAs, $n=12$ patients; one surgical intervention, $n=126$ patients; one surgery + radiotherapy, $n=7$ patients, one surgery+DAs, $n=68$ patients; one surgery + radiotherapy $+\mathrm{DAs}, n=21$ patients; two or more surgical interventions, $n=16$ patients; two or more surgeries + radiotherapy, $n=11$ patients; two surgeries + DAs, $n=32$ patients; and two or more surgeries + radiotherapy + DAs, $n=49$ patients. In Italy, a number of recent papers have described the treatment protocols $(10,11,12,13)$. During the study, 190 of the Bulgarian and 27 of the Italian patients were lost to follow-up and all of them had data relating to life/death status obtained from the civil registration and administrative services of both countries. Both baseline and follow-up GH and IGF1 samples were taken in the fasting state during routine hospital or outpatient assessment. Control of acromegaly in both centers after 1999 was defined as a basal GH level of $<2.5 \mathrm{ng} / \mathrm{ml}$ and/or a GH level of $<1 \mathrm{ng} / \mathrm{ml}$ during oral glucose tolerance test (OGTT) and normal age-adjusted IGF1 values (14). (When only one marker was present, remission was determined using it.) Both centers routinely 
used CT/MRI to assess the pituitary adenoma size. However, while Italy switched from CT to MRI at the beginning of the 1990s itself, this change was made during the mid-1990s in Bulgaria, which might constitute a bias in the precise assessment of the adenoma size between both cohorts. The date and cause of death were determined from general practice and hospital records, or from death certificates.

Diagnoses of hypertension and diabetes mellitus were set up according to internationally accepted criteria at the time of their diagnoses or as current use of an antihypertensive or a glucose-lowering drug was present. Blood pressure and carbohydrate metabolism were evaluated at each visit in both centers but patients' compliance to therapy has not been considered for the purpose of this study. Comorbidities represent the cumulative data captured over the entire follow-up period. The analysis of comorbidities was performed at the time of the last visit.

\section{Laboratory methods}

In the Naples laboratory, GH levels were assessed using modern IRMA or immunoenzymatic methods (sensitivity from 0.2 to $0.05 \mu \mathrm{g} / \mathrm{l}$ ). Serum IGF1 was measured by IRMA following ethanol extraction; normal ranges have been reported previously $(15,16)$. The assay sensitivity was $0.8 \mu \mathrm{g} / 1$. In Bulgaria, serum GH levels from 1992 onwards were determined by a solid-phase two-site fluorometric assay based on a direct sandwich technique with two monoclonal antibodies directed against two different epitopes of the human GH molecule (DELFIA; Perkin Elmer Life and Analytical Sciences, Wallac Oy, Finland). The sensitivity of this assay was $<0.03 \mathrm{mIU} / \mathrm{l}$. Until 1992, $\mathrm{GH}$ hormone values were measured by RIA. A conversion factor of two was used to transform the mIU/l units to $\mu \mathrm{g} / \mathrm{l}$. IGF1 levels were routinely assessed in the clinic from 1999 onwards. Serum IGF1 was measured using an IRMA following acid-alcohol extraction (Immunotech; Beckman Coulter Co., Marseille, France). The assay sensitivity was $<0.26 \mathrm{nmol} / \mathrm{l}$. Differences in IGF1 assay data were resolved using data transformation in the upper limit of normal (ULN) range $(\mathrm{ULN}=1)$.

Disturbances in any of the other pituitary axes were proven by appropriate biochemical tests or evidence of replacement therapy.

\section{Statistical analysis}

Results are expressed as mean \pm s.D. or as median (range) in normally and non-normally distributed data respectively.
Continuous data were analyzed using Student's $t$-test, the Mann-Whitney $U$ test when appropriate, and ANOVA analysis of more than two groups, while categorical data were analyzed using the $\chi^{2}$ test. Possible predictors of mortality were studied only in the Bulgarian cohort due to the small number of deaths in the Italian one. For this purpose, Cox regression analysis was used adjusted for age and sex, as the number of females was twice more than that of males. In order to assess their independent predictive power, significant or near-to-significant variables in the univariate analysis were applied in a multivariate Cox regression analysis. All statistical analyses were performed using SPSS for Windows version 16.0 (SPSS, Inc.). The mortality of both cohorts was compared with the general population using the standardized mortality ratio (SMR), based on reference mortality data adjusted for age, sex, calendar year, and region of residence, obtained from the statistical institutes of Bulgaria and Campania. The expected number was estimated by multiplying age-, sex-, and calendar period-specific death rates in the general population by the person-years at risk accumulated within the age-, sex-, and calendar period-specific strata corresponding to the patient cohort. Exact 95\% CIs and $P$ values were obtained using the normal approximation in which the S.E.M. of the natural logarithm of the SMR is one divided by the square root of the observed number of deaths for the CIs and the expected number of deaths for the $P$ value. Exact CIs were constructed and Poisson approximation for $P$ values was used when the observed number of deaths was $<100$. A $P$ value of $<0.05$ was considered statistically significant (17).

\section{Results}

\section{Characteristics of patients}

Table 1 summarizes all patients' characteristics and presents comparison between both cohorts. Owing to the introduction of IGF1 assay from 1999 onwards in the Bulgarian center, the analysis of IGF1 values of Bulgarian patients was performed only in those having available data at diagnosis: $13.8 \%$ compared with $83.6 \%$ of the Italian patients. IGF1 values at the last follow-up were available in 58.2 and $85.4 \%$ of the Bulgarian and Italian patients respectively.

\section{Treatment}

The most important difference in the treatment approach between both cohorts was the availability of SSAs and 
Table 1 Patients' characteristics.

\begin{tabular}{l}
\hline \\
\hline$n$ \\
Females \\
Mean age at diagnosis (years) \\
Median baseline GH levels ( $\mu$ g/l) \\
Median baseline IGF1 levels (ULN) \\
Duration from diagnosis \\
Years between symptoms and treatment \\
Number of deceased patients \\
Tumor size \\
Macroadenomas, $n$ (\%) \\
Comorbidities \\
Hypertension, $n$ (\%) \\
Diabetes, $n$ (\%) \\
Cancer, $n$ (\%) \\
Mean follow-up (years) \\
Median final GH levels ( $\mu$ g/l) \\
Median final IGF1 levels (ULN) \\
Treatment throughout the study period \\
Surgery, $n$ (\%) \\
Radiotherapy, $n$ (\%) \\
Somatostatin analogs (SSAs), $n(\%)$ \\
Dopamine agonists (DAs), $n(\%)$ \\
GH receptor antagonist, $n(\%)$ \\
Hypopituitarism \\
Hypopituitarism of at least one axis, $n(\%)$ \\
Panhypopituitarism, $n(\%)$ \\
Hypogonadism, $n(\%)$ \\
Hypothyroidism, $n(\%)$ \\
Hypoadrenalism, $n(\%)$ \\
\hline
\end{tabular}

\begin{tabular}{c}
\hline Bulgaria \\
\hline 407 \\
$268(65.8)$ \\
$41.8 \pm 11.34$ \\
$21.5(0.3-100.0)$ \\
$2.7(0.72-7.26)$ \\
$(n=56)$ \\
$13.73 \pm 8.6$ \\
$9.3 \pm 5.4$ \\
71
\end{tabular}

\begin{tabular}{|c|c|}
\hline Campania (Italy) & $\boldsymbol{P}$ (exact two-tailed) \\
\hline 220 & \\
\hline $112(50.9)$ & $<0.001$ \\
\hline $43.1 \pm 13.32$ & 0.22 \\
\hline $23.0(0.5-370)$ & 0.23 \\
\hline $\begin{array}{c}2.0(0.5-6.37) \\
(n=184)\end{array}$ & 0.001 \\
\hline $11.24 \pm 7.34$ & $<0.001$ \\
\hline $8.6 \pm 6.96$ & 0.176 \\
\hline 7 & $<0.001$ \\
\hline $175(79.6)$ & 0.16 \\
\hline $71(32.3)$ & $<0.001$ \\
\hline 47 (25.9) & 0.144 \\
\hline $10(4.5)$ & 0.375 \\
\hline $8.3 \pm 6.2$ & 0.014 \\
\hline $1.9(0 . \overline{1}-63.0)$ & $<0.001$ \\
\hline $\begin{array}{c}0.74(0.1-4.69) \\
(n=188)\end{array}$ & 0.006 \\
\hline $158(71.8)$ & 0.012 \\
\hline $34(15.5)$ & 0.003 \\
\hline $140(63.6)$ & $<0.001$ \\
\hline $87(39.5)$ & 0.205 \\
\hline $28(12.7)$ & $<0.001$ \\
\hline $43(19.5)$ & 0.182 \\
\hline $9(4.1)$ & 0.021 \\
\hline $53(24.1)$ & $<0.001$ \\
\hline $28(12.7)$ & 0.102 \\
\hline $27(12.3)$ & 0.89 \\
\hline
\end{tabular}

GHRAs. SSAs were used in Bulgaria during a clinical trial for a short period of time and none of the patients were under such treatment by the time of the last visit. Therefore, surgical interventions and radiotherapy were more frequently performed in Bulgaria due to insufficient funding for the respective medications (Table 1). Besides, the use of radiotherapy (telegammatherapy) in Bulgaria remained relatively high over the years. The mean total dose \pm s.D. was $45.3 \pm 7.6 \mathrm{~Gy}$. Only few patients had radiosurgery (gamma knife) that was performed out of the country. On the other hand, the use of radiotherapy in the Italian cohort sharply decreased after the introduction of SSAs in clinical practice, and conventional radioablation has been replaced by radiosurgery in most of the patients requiring radiotherapy. The use of DAs before the introduction of SSAs in clinical practice was comparable between the Campania and Bulgaria cohorts (39.5 vs $45.2 \%$; Table 1). However, this practice changed over time, such that administration of DAs decreased in $16 \%(n=35)$ of the Italian patients, the majority of whom used DAs in combination with SSAs or GHRAs, while it was still followed in the Bulgarian cohort. Moreover, it was documented that Bulgarian patients had a higher frequency of hypogonadism and total hypopituitarism.

Treatment outcomes are summarized in Table 2 . Significantly more patients in Campania have achieved normal IGF1 and safe GH levels at the last visit mainly due to the beneficial effect of the medical treatment. As no Bulgarian patient has been treated with SSAs or GHRAs at the last follow-up, we assume that this beneficial effect is related to the greater efficacy of the latter two.

\section{Mortality data}

Over the study period, deceased patients were significantly less in Campania (7) than in Bulgaria (71) by absolute numbers $(P<0.001)$ (Fig. 1). Moreover, the observed/ expected ratio in Bulgaria was significantly higher compared with the general population, unlike the Italian one: $71 / 35.4$ (95\% CI $1.54-2.47 ; P<0.05$ ) vs $7 / 10.6$ (95\% CI $0.27-1.36 ; P=\mathrm{NS})$. Male/female ratio was 0.75 and 0.65 $(P=1.00)$ in Campania and Bulgaria respectively; mean 
Table 2 Treatment outcomes at the last visit in Bulgaria and Campania.

\begin{tabular}{|c|c|c|c|c|c|c|c|c|c|}
\hline & \multicolumn{3}{|c|}{ Safe GH levels ${ }^{a}$} & \multicolumn{3}{|c|}{ IGF1 $($ ULN) $\leq 1.0$} & \multicolumn{3}{|c|}{ Remission combined $^{b}$} \\
\hline & Bulgaria & Italy & $P$ value & Bulgaria & Italy & $P$ value & Bulgaria & Italy & $P$ value \\
\hline Surgery, n/total (\%) & $96 / 165(58.2)$ & $27 / 42(64.2)$ & 0.598 & $75 / 108$ (69.4) & 24/33 (72.7) & 0.829 & $95 / 165(57.6)$ & $28 / 45(62.2)$ & 0.612 \\
\hline $\begin{array}{l}\text { Rö without S, } \\
\text { n/total (\%) }\end{array}$ & $8 / 18(44.4)$ & $2 / 5(40)$ & 1.0 & $2 / 5(40)$ & $3 / 3(100)$ & 0.196 & $8 / 18(44.4)$ & $2 / 5(40)$ & 1.0 \\
\hline $\mathrm{S}+\mathrm{M}, n /$ total $(\%)$ & $22 / 81(27.2)$ & $42 / 79(53.2)$ & 0.001 & $20 / 63(31.7)$ & $51 / 80(63.8)$ & $<0.001$ & $14 / 81(17.2)$ & $41 / 84(48.8)$ & $<0.001$ \\
\hline S+Rö, n/total (\%) & $24 / 39(61.5)$ & $11 / 14(78.5)$ & 0.333 & $18 / 25(72)$ & $8 / 10(80)$ & 1.0 & $24 / 41(58.5)$ & $11 / 14(78.5)$ & 0.215 \\
\hline $\mathrm{S}+\mathrm{Rö}+\mathrm{M}$, n/total (\%) & $14 / 42(33.3)$ & $9 / 15(60)$ & 0.124 & $20 / 36(55.6)$ & $11 / 14(78.6)$ & 0.197 & $12 / 42(28.6)$ & $8 / 15(53.3)$ & 0.117 \\
\hline M only, n/total (\%) & 2/12 (16.7) & $21 / 45(46.7)$ & 0.103 & $2 / 6(33.3)$ & $33 / 45(73.3)$ & 0.069 & 4/13 (30.8) & $22 / 47(46.8)$ & 0.358 \\
\hline Others, n/total (\%) & $11 / 47(23.4)$ & $3 / 5(60)$ & 0.114 & 2/15 (13.3) & $2 / 3(66.7)$ & 0.108 & $2 / 47(4.3)$ & $0 / 10(0)$ & 1.0 \\
\hline Total, n/total (\%) & $177 / 404(43.8)$ & $115 / 205(56.1)$ & 0.005 & $139 / 258(53.9)$ & $132 / 188(70.2)$ & 0.001 & $159 / 407(39.1)$ & $112 / 220(50.9)$ & 0.005 \\
\hline
\end{tabular}

Rö, radiotherapy; M, medical therapy; S, surgery; Others, newly diagnosed patients without further information on treatment or patients without treatment. ${ }^{a}$ Safe GH levels were defined as basal GH levels of $<2.5 \mu \mathrm{g} / \mathrm{l}$ and/or GH levels of $<1 \mu \mathrm{g} / \mathrm{l}$ during OGTT.

${ }^{\mathrm{b}}$ Remission is defined by both safe GH and normal IGF1 values. If one of the variables was missing, remission was defined by the present one.

age on diagnosis was higher in Campania without reaching statistical significance $(53.7 \pm 16.6$ vs $46.4 \pm 11.5$; $P=0.129)$. Mean age of death was similar between both countries: $61.3 \pm 13.2$ vs $61.9 \pm 11.0(P=0.885)$ for Campania and Bulgaria respectively. In Campania, 3/2.5 (observed/expected) patients died due to cardiovascular causes (one myocardial infarction and two heart failures), 3/5.7 due to neoplasms (uterus and pituitary tumor), and $1 / 8$ due to other causes (nutritional disorder). In Bulgaria, 21/5.9 (observed/expected) patients died due to cerebrovascular causes (coded as stroke in 19 and chronic cerebrovascular disease in two), 31/13.4 patients due to cardiovascular causes (acute myocardial infarction in ten, chronic ischemic heart disease in six, heart failure in five, pulmonary thromboembolism in five, cardiac arrest in four, and cardiomyopathy in one), 12/8.4 patients due to malignant tumors (two cases with lung cancer; two with hepatic cancer; and one with prostate, breast, coli uteri, ovarian, cerebral, pancreatic, and colon cancers, and a melanoma), and 7/8.2 due to other causes (renal infection, COPD, pneumonia, chronic renal failure, diabetes mellitus, disease of the digestive tract, and nontoxic goiter).

As mentioned above, the estimated mortality of the Italian cohort, expressed as SMR, was similar to that of the general population, while Bulgarian patients had two times higher mortality rates compared with the general population. Patients in Bulgaria had significantly increased cerebrovascular and cardiovascular death rates (Table 3). Both irradiated and nonirradiated patients had an increased mortality with a predominance of cerebrovascular causes in irradiated and cardiovascular causes in nonirradiated patients. All-cause and vascular mortalities were restored to normal in patients in remission. However, the interpretation of cerebrovascular mortality in remission should be cautious due to the small number of deaths.

\section{Effect of biochemical control and other factors on mortality}

Factors predicting mortality in the Bulgarian cohort in univariate Cox regression analysis (adjusted for age and sex) were levels of GH and IGF1 at the last visit, duration of active disease, uncontrolled comorbidities (arterial hypertension, diabetes mellitus, and dyslipidemia), and patients lost to follow-up (Table 4). When these variables were applied in a multivariate analysis, age at diagnosis and last $\mathrm{GH}$ value remained as the independent predictors of mortality. IGF1 was excluded from the multivariate

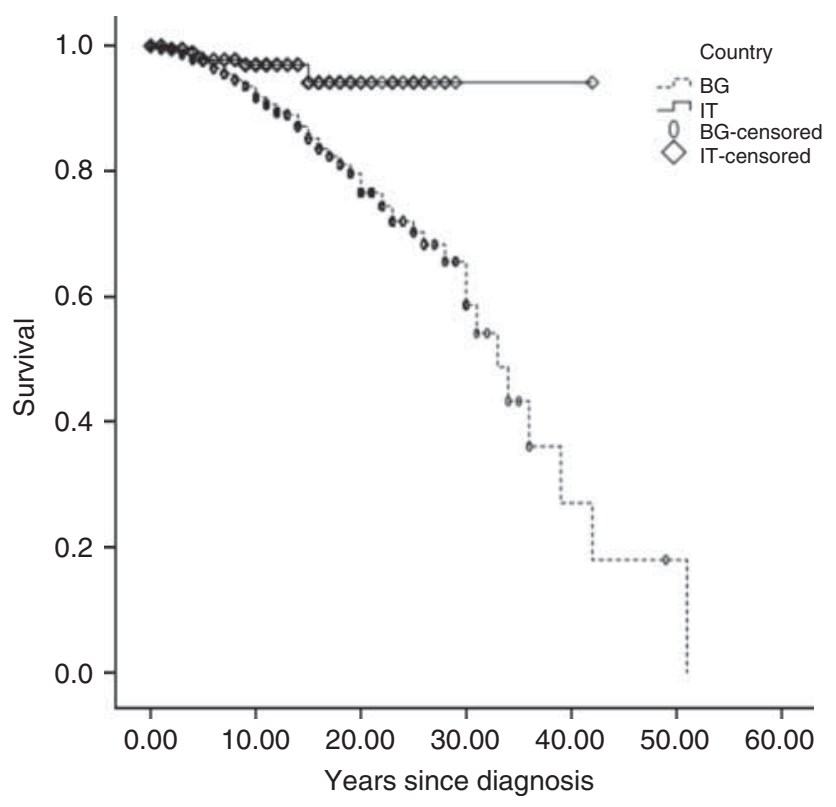

Figure 1

Kaplan-Meier survival analysis in the Bulgarian and the Italian cohorts. 
Table 3 All-cause and cause-specific mortality of the Bulgarian cohort.

All-cause
All-cause in remission
Cardiovascular
Cardiovascular in remission
Cerebrovascular
Cerebrovascular in remission
Neoplastic
Other causes
All-cause irradiated
Irradiated in remission
Nonirradiated
Nonirradiated in remission
Cerebrovascular in irradiated
Cerebrovascular in nonirradiated
Cardiovascular in irradiated
Cardiovascular in nonirradiated

\begin{tabular}{c}
\hline Observed \\
\hline 71 \\
19 \\
31 \\
6 \\
21 \\
5 \\
12 \\
7 \\
23 \\
7 \\
48 \\
11 \\
11 \\
10 \\
7 \\
24 \\
\hline
\end{tabular}

\begin{tabular}{c}
\hline Expected \\
\hline 35.4 \\
15.2 \\
13.4 \\
5.7 \\
5.9 \\
2.5 \\
8.4 \\
8.2 \\
8.9 \\
3.2 \\
27 \\
11.5 \\
1.5 \\
4.4 \\
3.4 \\
10.1 \\
\hline
\end{tabular}

\begin{tabular}{l}
\hline SMR \\
\hline 2.00 \\
1.25 \\
2.31 \\
1.05 \\
3.54 \\
1.98 \\
1.43 \\
0.85 \\
2.59 \\
2.21 \\
1.77 \\
0.96 \\
7.15 \\
2.28 \\
2.08 \\
2.38 \\
\hline
\end{tabular}

\begin{tabular}{c}
\hline $\mathbf{9 5 \%} \mathbf{~ C I}$ \\
\hline $1.54-2.47$ \\
$0.69-1.81$ \\
$1.49-3.13$ \\
$0.21-1.89$ \\
$2.03-5.06$ \\
$0.24-3.72$ \\
$0.62-2.24$ \\
$0.22-1.49$ \\
$1.53-3.65$ \\
$0.57-3.84$ \\
$1.27-2.28$ \\
$0.38-1.52$ \\
$2.92-11.37$ \\
$0.87-3.69$ \\
$0.54-3.62$ \\
$1.43-3.34$ \\
\hline
\end{tabular}

\begin{tabular}{cc}
\hline $\boldsymbol{P}$ \\
\hline$<0.05$ \\
NS \\
$<0.05$ \\
NS \\
$<0.05$ \\
NS \\
NS \\
NS \\
$<0.05$ \\
NS \\
$<0.05$ \\
NS \\
$<0.05$ \\
NS \\
NS \\
$<0.05$ \\
\hline
\end{tabular}

analysis due to the small number of deceased patients with available data $(n=14)$ and consequently highly unreliable statistical results. A separate multivariate Cox regression model was built using the GH values before irradiation. $\mathrm{GH}$ values and age at diagnosis were significant predictors of mortality (hazard ratio (HR) 1.024 (95\% CI 1.0011.047), $P=0.037$ and HR 1.2 (95\% CI 1.11-1.30), $P<0.001)$, respectively, while lost to follow-up or control of arterial hypertension, diabetes, and dyslipidemia did not show any statistical significance. There was a trend for a higher rate of deaths in patients after unsuccessful surgery, $14.6 \%(34 / 233)$, vs cured patients after operation, $7.3 \%$ (7/96), $P=0.097$. However, the Cox model did not show any significant influence of surgical outcome on mortality. Patients who were followed up regularly had a significantly higher percentage of hypopituitarism compared with patients who were lost to follow-up, 41.8 vs $24.4 \%$ respectively. As the actual rate of hypopituitarism by the end of the study period in the latter could not be estimated, the influence of hypopituitarism was studied in only regularly followed-up patients. Patients lost to follow-up were censored in the analysis of all the other variables. After being adjusted for age, none of the deficient axes were associated with higher HRs: hypogonadism HR 1.84 (95\% CI 0.46-7.4; $P=0.389$ ); hypothyroidism HR 5.12 (95\% CI 0.66-39.95; $P=0.119$ ); and hypoadrenalism HR 2.5 (95\% CI 0.32-19.42; $P=0.38$ ). Panhypopituitarism vs no pituitary deficiency was not

Table 4 Effect of biochemical and other factors on mortality in acromegaly patients.

\begin{tabular}{|c|c|c|c|c|c|c|}
\hline & \multicolumn{3}{|c|}{$\begin{array}{l}\text { Univariate sex- and age-adjusted Cox } \\
\text { regression analysis }\end{array}$} & \multicolumn{3}{|c|}{ Multivariate Cox regression analysis } \\
\hline & $\mathrm{HR}$ & $95 \% \mathrm{Cl}$ & $P$ & $\mathrm{HR}$ & $95 \% \mathrm{Cl}$ & $P$ \\
\hline Gender (male) & 0.68 & $0.41-1.13$ & 0.14 & & & \\
\hline Age at diagnosis (years) & 1.10 & $1.07-1.13$ & $<0.001$ & 1.11 & $1.08-1.15$ & $<0.001$ \\
\hline Last GH $(\mu \mathrm{g} / \mathrm{l})$ & 1.02 & $1.01-1.03$ & $<0.001$ & 1.02 & $1.007-1.03$ & 0.001 \\
\hline Last IGF1 (nmol/l) & 1.01 & $1.01-1.03$ & $<0.001$ & & & \\
\hline $\mathrm{GH}$ at diagnosis & 1.002 & $0.99-1.01$ & 0.541 & & & \\
\hline Adenoma size at diagnosis (macroadenoma) & 1.31 & $0.76-2.26$ & 0.33 & & & \\
\hline Radiotherapy (yes vs no) & 1.51 & $0.89-2.58$ & 0.128 & & & \\
\hline Cured after surgery (no vs yes) & 1.12 & $0.48-2.62$ & 0.798 & & & \\
\hline Hypopituitarisma (yes vs no) & 2.32 & $0.68-7.91$ & 0.137 & & & \\
\hline Hypertension $^{\mathrm{b}}$ (controlled vs uncontrolled) & 2.20 & $1.27-3.82$ & 0.005 & 0.87 & $0.45-1.67$ & 0.673 \\
\hline Diabetes $^{b}$ (controlled vs uncontrolled) & 3.03 & $1.54-5.93$ & 0.001 & 0.79 & $0.35-1.76$ & 0.557 \\
\hline 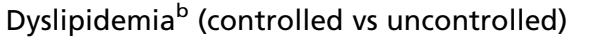 & 1.84 & $1.06-3.2$ & 0.031 & 0.695 & $0.38-1.27$ & 0.237 \\
\hline Lost to follow-up (yes vs no) & 2.42 & $1.31-4.45$ & 0.005 & 0.99 & $0.45-2.21$ & 0.983 \\
\hline
\end{tabular}

${ }^{a}$ Only regularly followed-up patients were analyzed.

${ }^{b}$ Controlled includes also patients without hypertension, diabetes, or dyslipidemia, respectively. 
associated with a significantly higher mortality ratio (HR 2.002 (95\% CI 0.26-15.54); $P=0.51$ ).

As SMR for cerebrovascular death was increased in irradiated patients, we have additionally studied the effect of radiotherapy in this subset of the cohort. In a univariate analysis corrected for age and sex, radiotherapy was a negative predictor of survival with a HR of 4.54 (95\% CI 1.54-11.68), $P=0.005$. In a multivariate analysis including age at diagnosis, $\mathrm{GH}$ values at last visit, lost to follow-up, arterial hypertension, diabetes, and dyslipidemia, irradiation remained an independent predictor of mortality with a HR of 5.75 (95\% CI 1.75-19.2) $P=0.004$, together with age at diagnosis with a HR of 1.15 (95\% CI 1.08-1.22), $P<0.001$, a trend in the last $\mathrm{GH}$ value with a HR of 1.02 (95\% CI 0.997-1.04), $P=0.093$, and no statistically significant influence of the other variables.

In order to study the influence of different treatment modalities, patients were divided into three groups: group 1 (treated by surgery, $n=165 ;$ ), group 2 (treated with adjuvant to surgery medical therapy (DAs), $n=81$ ), and group 3 (operated and irradiated $+/-$ medical therapy, $n=83$ ) (Table 5).

Lost to follow-up vs regularly followed-up patients had significantly higher percentages of uncontrolled arterial hypertension (60\% (48/80) vs 23.7\% (32/135), $P<0.001)$, dyslipidemia (83.2\% (79/95) vs 63.3\% (57/90), $P=0.003)$, and comparable control of diabetes $(35.9 \%$ $(14 / 39)$ vs $26.6 \%(17 / 64), P=0.378)$. Active vs patients in remission had comparable percentages of uncontrolled comorbidities diabetes (31.6\% (24/76) vs 25.9\% (7/27), $P=0.634)$, arterial hypertension (36.5\% (46/126) vs $38.2 \%$ (34/89), $P=0.886)$, and dyslipidemia $(68.2 \%(73 / 107)$ vs $80.8 \%(63 / 78), P=0.065)$.

\section{Discussion}

This was a retrospective, comparative study of two acromegaly cohorts, in Bulgaria and Campania region in Italy, aiming at assessing the mortality during the period of 1999-2008 as related to different treatment modalities and control of acromegaly.

Since the first series of Wright et al. (18) in 1970 assessing the mortality, a number of studies have demonstrated that acromegaly is associated with a reduced life expectancy due to cardiovascular, cerebrovascular, respiratory diseases, and malignancy in different proportions (19). In accordance with our results of the Bulgarian cohort, increased vascular mortality has been reported in several studies $(20,21,22,23,24)$. On the other hand, a tendency toward increased life expectancy has been observed in recent studies $(22,23,24,25,26,27$, $28,29,30,31,32,33,34)$. They suggested that the improved survival rate could be due to more stringent criteria for remission and the introduction of modern and more efficient treatment options, such as SSAs and GHRAs. A recent meta-analysis by Holdaway et al. (7) has demonstrated that studies with $>30 \%$ utilization of SSAs or $>70 \%$ of remission rates reported normal life expectancy in contrast to higher mortality rates in those with lower percentages of SSA administration and remission rates. In line with these findings, the Campania cohort had a normal life expectancy - an SMR of 0.66 (95\% CI $0.27-1.36 ; P=0.269)$ in agreement with two recent studies concerning mortality among the Italian population (33, 34, 35). Owing to funding restrictions in Bulgaria, SSAs and GHRAs were not available in clinical practice until 2008 and none of the patients were under such

Table 5 Characteristics of patients in the three treatment groups. Significance was assessed using ANOVA analysis in continuous variables and Fisher's exact test in categorical variables.

\section{Variables}

Females, $n(\%)$

Age at diagnosis (years)

Macroadenoma at diagnosis $n(\%)$

Last GH (mIU/l)

Last IGF1 ( $\mathrm{nmol} / \mathrm{l})$

Duration of follow-up (years)

Hypertension (absence or controlled), $n(\%)$

Diabetes (absence or controlled), $n(\%)$

Dyslipidemia (absence or controlled), $n(\%)$

Deceased patients, $n(\%)$

Age at death (years)

Lost to follow-up, $n(\%)$

\begin{tabular}{c}
\hline Surgery $(n=165)$ \\
\hline $99(60)$ \\
$44.1 \pm 10.3$ \\
$95(61.3)$ \\
$12.69 \pm 20.55$ \\
$39.2 \pm 33.04$ \\
$11.6 \pm 7.2$ \\
$119(78 \%)$ \\
$148(94.2)$ \\
$92(62.1)$ \\
$25(15.2)$ \\
$57.0 \pm 9.6$ \\
$84(50.9)$
\end{tabular}

\begin{tabular}{c}
\hline $\mathbf{S}+\mathbf{M}(n=81)$ \\
\hline $54(66.7)$ \\
$39.5 \pm 12.9$ \\
$64(79)$ \\
$18.47 \pm 22.64$ \\
$56.97 \pm 30.76$ \\
$12.6 \pm 8.0$ \\
$69(86.3 \%)$ \\
$71(87.7)$ \\
$56(71.8)$ \\
$6(7.4)$ \\
$56.0 \pm 12.9$ \\
$23(28.4)$ \\
\hline
\end{tabular}

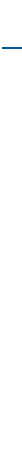

$\begin{array}{r}r \text { P value } \\ \hline 0.027 \\ <0.001 \\ <0.001 \\ 0.101 \\ 0.006 \\ <0.001 \\ 0.298 \\ 0.159 \\ 0.147 \\ 0.229 \\ 0.957 \\ <0.001 \\ \hline\end{array}$

$\mathrm{S}+\mathrm{M}$, surgery plus medical treatment; $\mathrm{S}+\mathrm{Rö}$, surgery plus radiotherapy $+/-$ medical therapy. 
treatment at the last visit. Bulgarian patients had access only to DAs (bromocriptine and cabergoline) known for their lower efficacy in acromegaly $(36,37)$. The lack of SSAs and GHRAs and the poorer efficacy of DAs are the most possible explanation for the significantly higher rates of other treatment options, such as neurosurgical interventions and radiotherapy. These differences in the treatment approach resulted in a significantly better biochemical control (safe GH and normal IGF1 levels) of acromegaly in Campania, mainly due to adjuvant medical therapy (Table 2). As expected, the mortality was significantly higher in Bulgarian vs Campania patients when evaluated by absolute numbers (71 vs 7 deceased patients; $P<0.001$ ) (Fig. 1) and compared with both general populations (Table 3).

The higher all-cause and vascular mortality in the Bulgarian cohort were restored to normal in patients in remission with an SMR of 1.25 (95\% CI 0.69-1.81) for all-cause and SMR of 1.05 (95\% CI 0.21-1.89) for cardiovascular deaths. Cerebrovascular death was also without any significant difference with respect to the general population, although SMR was almost two times higher at 1.98 (95\% CI 0.24-3.75, $P=\mathrm{NS})$. The latter result should be interpreted with caution, as the number of deaths was relatively small. The beneficial effect of biochemical control on the outcome has been widely investigated. Bates et al. (38) were the first to demonstrate that an increased mortality was reduced to normal when GH levels reached $<2.5 \mu \mathrm{g} / \mathrm{l}$. Their observations were supported by numerous further studies $(22,26,27,28,29$, $30,32,38,39)$ with a pooled SMR of $1.1(0.9-1.4 ; P=0.50)$ (7). Some research groups reported even lower GH targets: $2 \mu \mathrm{g} / \mathrm{l}$ by Ayuk et al. and a further improvement in mortality when GH was reduced to $<1 \mu \mathrm{g} / 1(23,26)$. Indeed, the last GH value (or post-glucose GH (30)) has been ascertained as a strong predictor of mortality by uniand multivariate analyses in various surveys $(23,26,27$, $33,35)$. In agreement, the last $\mathrm{GH}$ value was an independent predictor of mortality in our study as well (Table 4). Mortality rates were restored to levels comparable to the general population when IGF1 values were also below the ULN $(25,26,30,39)$. Although the majority of patients with normal IGF1 values had safe GH levels, the independent role of the growth factor for survival was not demonstrated in such a definite manner $(23,26,27$, $33,35)$. A probable explanation for such controversial results could be the relatively smaller number of patients tested for IGF1, methodological difficulties in IGF1 measurements, as well as the lack of own age-adjusted reference values. In the Bulgarian cohort, IGF1 was a negative predictor of survival in the univariate analysis but due to the small number of deceased patients with available IGF1 values, its role as an independent predictor of mortality could not be reliably studied.

Apart from factors specific for acromegaly, other classical risk factors influencing particularly vascular mortality, such as hypertension, diabetes, and dyslipidemia, might influence the life expectancy. Control of these comorbidities in the Bulgarian cohort significantly improved the survival prognosis according to the univariate Cox model. Only few surveys have demonstrated an independent predictive role of diabetes and/or hypertension $(18,20,26)$. Cardiovascular and glucose metabolism alterations are known to be more frequent in patients with acromegaly and dependent on disease control (6). This interrelation could be a possible explanation for the observation that comorbidities have lost their significant influence in multivariate analysis where biochemical status has preserved its dominant role $(2,33,35,38)$. In agreement, in our study, hypertension, diabetes mellitus, and dyslipidemia were not independent predictors of mortality in the multivariate analysis. However, comorbidities could not be neglected, because there are no studies to properly investigate the complex interrelation between their management and outcomes in acromegaly as yet. Furthermore, as acromegaly is a rare disease, the number of censored patients always remains relatively small, which could question the reliability of the statistical results.

With regard to the specific treatment approach, the detrimental effect of radiotherapy has been seriously studied in the last decade $(40,41)$. Thus far, four studies have presented SMR in irradiated vs nonirradiated patients with acromegaly and all of them have demonstrated significantly increased death rates with respect to radiotherapy, mainly due to higher cerebrovascular death rates $(23,24,27,34)$. Radiotherapy has also been proved to be an independent predictor of mortality $(23,24,31,33,35)$. In our study, both irradiated and nonirradiated patients had a higher all-cause mortality compared with the general population, with SMRs of 2.59 (95\% CI 1.53-3.65) and 1.77 (95\% CI 1.27-2.28) respectively. However, telegammatherapy was associated with seven times higher cerebrovascular death rate than expected - an SMR of 7.15 (95\% CI 2.92-11.37) while other vascular mortalities were not significantly increased (Table 3). Regression analysis revealed similar data - radiotherapy was a strong independent predictor of cerebrovascular mortality. Direct comparison with other studies with regard to the all-cause mortality could be hampered by different 
proportions of death causes and different time periods between irradiation and censoring.

Patients who underwent surgery as the only treatment had a better biochemical profile at the time of the last follow-up; however, the number of deceased patients was similar compared with those treated with medical therapy and/or radiotherapy. Probably, some other factors have influenced the outcome, which we could not study due to the small number of deceased patients in the groups. The relatively low cure rates after surgery (29\% overall) could have also been a factor impeding a manifestation of beneficial influence of successful surgery on mortality. With regard to treatment options, a recent survey has shown for the first time a higher death risk in acromegaly patients with diabetes mellitus treated with primary SSAs despite appropriate correction for several covariates (35). Owing to the small number of patients on primary medical therapy (DAs) in the Bulgarian population, its effect on mortality has not been examined.

Tumor expansion and especially time after radiotherapy are associated with an increased risk for hypopituitarism in acromegaly (19). Hypopituitarism has been related to an increased mortality in nonfunctioning pituitary adenomas although its independent role for decreased survival in acromegaly is disputable (40). Most of the studies did not demonstrate association or predictive role of pituitary deficiency in either of the axes $(26,31,33,34)$. In agreement, hypopituitarism was not related to a higher mortality in the Bulgarian cohort. However, the analysis of patients from the West Midland Database showed an increased mortality risk in patients with ACTH deficiency and especially those on higher doses of hydrocortisone ( $>25 \mathrm{mg}$ daily), explained by mechanisms similar to adrenal deficiency and Cushing's syndrome (24). Patients with hypopituitarism had an increased mortality rate close to significance in a multivariate model with IGF1 in the study by Bogazzi et al. (35).

Undoubtedly, the current study has several weak points. The period of evaluation of mortality is shorter than the period of follow-up, which could create a bias in our results. However, acromegaly is a rare disease and evaluation of 10-year mortality since diagnosis would not provide a sufficient number of both alive and deceased patients to draw reliable conclusions. On the other hand, assessment of mortality for a longer period of time could have been hampered by the lack of reliable normative mortality data of the general population or life/death status of all consecutive patients. Inclusion of patient-years before modern treatment in Italy was available could also bias our findings. External analysis (comparison between the study and general population) and, moreover, comparison between two different study cohorts originating from different general populations has its pitfalls as there could be a number of difficult-to-measure variables. In line with this, Bulgarian and Italian populations have some differences in cardiovascular risk factor burden that should be taken into consideration when speculating over the mortality rates of both cohorts. By the end of the study (2008), the prevalence of hypertension, obesity, and smoking was higher in Bulgaria than in Italy: 51.4 vs $46.1 \%$; 23.7 vs $19.1 \%$; and 34.3 vs $19.6 \%$ respectively (www.who.int/nmh/countries/), while the mean total cholesterol and fasting glucose levels were of comparable values. Standardized death rate per 100000 for diseases of the circulatory system was several times higher in Bulgaria (611.28) than in Italy (173.8) (www.data.euro.who.int/ hfadb/). Furthermore, apart from the treatment approach and treatment outcomes, both patient cohorts had some baseline differences (Table 1). We have tried to overcome the differences between cohorts by performing internal analysis of factors influencing mortality in the Bulgarian cohort. Clearly, the retrospective nature of the study might have biased data collection of comorbidities and this deserves a carefully designed prospective study to better understand the power of individual comorbidity and its treatment on mortality in acromegaly.

\section{Conclusion}

This retrospective study compared the mortality rates during the period of 1999-2008 of two acromegaly cohorts followed up for over a 40-year period of time. The more frequent adjuvant medical therapy and especially the use of SSAs and GHRA in Campania, as well as leading to a decreased use of radiotherapy, was the major difference in treatments between both patient populations. The integrated approach resulted in a better disease control of the Italian patients. Bulgarian patients had an increased vascular mortality and, as a result of relatively high use of conventional radiotherapy by modern standards, an increased cerebrovascular death rate. Although both patient cohorts originate from populations with different risk factor backgrounds, disease activity in this study was demonstrated to be a major predictor of mortality. In line with this, we speculate that modern treatment options by reducing hormone levels could increase the life expectancy. Nevertheless, due to the high circulatory death rates of the general population in Bulgaria, the influence of comorbidities, such as hypertension, diabetes, and dyslipidemia, could not be thoroughly neglected. The additional beneficial effect 
of comorbidities' management remains to be elucidated in future prospective studies.

\section{Declaration of interest}

This study did not receive any financial support. During the study period, A Colao was recipient of unrestricted grants by Ipsen, Italfarmaco, Novartis, and Pfizer for research programs in acromegaly, received lecture fees from Ipsen, Italfarmaco, Novartis, and Pfizer, and has been a member of the Scientific Boards of Novartis and Ipsen. R Pivonello received lecture fees from Novartis. S Zacharieva received lecture fees for lectures on acromegaly from Novartis and Pfizer. None of the other authors have any conflict of interest to disclose.

\section{Funding}

This research did not receive any specific grants from any funding agency in the public, commercial, or not-for-profit sector.

\section{References}

1 Alexander L, Appleton D, Hall R, Ross WM \& Wilkinson R. Epidemiology of acromegaly in the Newcastle region. Clinical Endocrinology 1980 12 71-79. (doi:10.1111/j.1365-2265.1980.tb03135.x)

2 Bengtsson BA, Eden S, Ernest I, Oden A \& Sjogren B. Epidemiology and long-term survival in acromegaly. A study of 166 cases diagnosed between 1955 and 1984. Acta Medica Scandinavica 1988223 327-335. (doi:10.1111/j.0954-6820.1988.tb15881.x)

3 Daly AF, Rixhon M, Adam C, Dempegioti A, Tichomirowa MA \& Beckers A. High prevalence of pituitary adenomas: a crosssectional study in the province of Liege, Belgium. Journal of Clinical Endocrinology and Metabolism 200691 4769-4775. (doi:10.1210/jc.2006-1668)

4 Fernandez A, Karavitaki N \& Wass JA. Prevalence of pituitary adenomas: a community-based, cross-sectional study in Banbury (Oxfordshire, UK). Clinical Endocrinology 201072 377-382. (doi:10.1111/j.1365-2265. 2009.03667.x)

5 Melmed S, Colao A, Barkan A, Molitch M, Grossman AB, Kleinberg D, Clemmons D, Chanson P, Laws E, Schlechte J et al. Guidelines for acromegaly management: an update. Journal of Clinical Endocrinology and Metabolism 200994 1509-1517. (doi:10.1210/jc.2008-2421)

6 Colao A, Ferone D, Marzullo P \& Lombardi G. Systemic complications of acromegaly: epidemiology, pathogenesis, and management. Endocrine Reviews 200425 102-152. (doi:10.1210/er.2002-0022)

7 Holdaway IM, Bolland MJ \& Gamble GD. A meta-analysis of the effect of lowering serum levels of GH and IGF-I on mortality in acromegaly. European Journal of Endocrinology 2008159 89-95. (doi:10.1530/ EJE-08-0267)

8 Colao A, Auriemma RS, Lombardi G \& Pivonello R. Resistance to somatostatin analogs in acromegaly. Endocrine Reviews 201032 247-271. (doi:10.1210/er.2010-0002)

9 Vandeva S, Andreeva M, Orbetsova M, Charakciev D, Kirilov G, Elenkova A, Atanasova I, Natchev E, Yaneva M, Kumanov P et al. Acromegaly in Bulgaria - epidemiological characteristics derived from the National Acromegaly Database. Endocrinolgia 201015 142-150.

10 Colao A, Martino E, Cappabianca P, Cozzi R, Scanarini M \& Ghigo E. First-line therapy of acromegaly: a statement of the A.L.I.C.E, (Acromegaly primary medical treatment Learning and Improvement with Continuous Medical Education) Study Group. Journal of Endocrinological Investigation 200629 1017-1020. (doi:10.1007/ BF03349217)
11 Colao A, Auriemma RS, Lombardi G \& Pivonello R. Resistance to somatostatin analogs in acromegaly. Endocrine Reviews 201132 247-271. (doi:10.1210/er.2010-0002)

12 Colao A, Auriemma RS, Galdiero M, Cappabianca P, Cavallo LM, Esposito F, Grasso LF, Lombardi G \& Pivonello R. Impact of somatostatin analogs versus surgery on glucose metabolism in acromegaly: results of a 5-year observational, open, prospective study. Journal of Clinical Endocrinology and Metabolism 200994 528-537. (doi:10.1210/jc.2008-1546)

13 Colao A, Pivonello R, Galderisi M, Cappabianca P, Auriemma RS, Galdiero M, Cavallo LM, Esposito F \& Lombardi G. Impact of treating acromegaly first with surgery or somatostatin analogs on cardiomyopathy. Journal of Clinical Endocrinology and Metabolism 200893 2639-2646. (doi:10.1210/jc.2008-0299)

14 Giustina A, Barkan A, Casanueva FF, Cavagnini F, Frohman L, Ho K, Veldhuis J, Wass J, Von Werder K \& Melmed S. Criteria for cure of acromegaly: a consensus statement. Journal of Clinical Endocrinology and Metabolism 200085 526-529. (doi:10.1210/jcem.85.2.6363)

15 Colao A, Spiezia S, Di Somma C, Pivonello R, Marzullo P, Rota F, Musella T, Auriemma RS, De Martino MC \& Lombardi G. Circulating insulin-like growth factor-I levels are correlated with the atherosclerotic profile in healthy subjects independently of age. Journal of Endocrinological Investigation 200528 440-448. (doi:10.1007) BF03347225)

16 Colao A, Di Somma C, Cascella T, Pivonello R, Vitale G, Grasso LF, Lombardi G \& Savastano S. Relationships between serum IGF1 levels, blood pressure, and glucose tolerance: an observational, exploratory study in 404 subjects. European Journal of Endocrinology 2008159 389-397. (doi:10.1530/EJE-08-0201)

17 Goldblatt P. Longitudinal study: mortality and social organisation, vol 6. Series LS; HMSO London, 1990.

18 Wright AD, Hill DM, Lowy C \& Fraser TR. Mortality in acromegaly. Quarterly Journal of Medicine 197039 1-16.

19 Sherlock M, Ayuk J, Tomlinson JW, Toogood AA, Aragon-Alonso A, Sheppard MC, Bates AS \& Stewart PM. Mortality in patients with pituitary disease. Endocrine Reviews 201031 301-342. (doi:10.1210/ er.2009-0033)

20 Rajasoorya C, Holdaway IM, Wrightson P, Scott DJ \& Ibbertson HK. Determinants of clinical outcome and survival in acromegaly. Clinical Endocrinology 199441 95-102. (doi:10.1111/j.1365-2265.1994. tb03789.x)

21 Etxabe J, Gaztambide S, Latorre P \& Vazquez JA. Acromegaly: an epidemiological study. Journal of Endocrinological Investigation 199316 181-187. (doi:10.1007/BF03344942)

22 Orme SM, McNally RJ, Cartwright RA \& Belchetz PE. Mortality and cancer incidence in acromegaly: a retrospective cohort study. United Kingdom Acromegaly Study Group. Journal of Clinical Endocrinology and Metabolism 199883 2730-2734. (doi:10.1210/jcem. 83.8.5007)

23 Ayuk J, Clayton RN, Holder G, Sheppard MC, Stewart PM \& Bates AS. Growth hormone and pituitary radiotherapy, but not serum insulinlike growth factor-I concentrations, predict excess mortality in patients with acromegaly. Journal of Clinical Endocrinology and Metabolism 2004 89 1613-1617. (doi:10.1210/jc.2003-031584)

24 Sherlock M, Reulen RC, Alonso AA, Ayuk J, Clayton RN, Sheppard MC, Hawkins MM, Bates AS \& Stewart PM. ACTH deficiency, higher doses of hydrocortisone replacement, and radiotherapy are independent predictors of mortality in patients with acromegaly. Journal of Clinical Endocrinology and Metabolism 200994 4216-4223. (doi:10.1210/ jc.2009-1097)

25 Swearingen B, Barker FG II, Katznelson L, Biller BM, Grinspoon S, Klibanski A, Moayeri N, Black PM \& Zervas NT. Long-term mortality after transsphenoidal surgery and adjunctive therapy for acromegaly. Journal of Clinical Endocrinology and Metabolism 199883 3419-3426. (doi:10.1210/jcem.83.10.5222) 
26 Holdaway IM, Rajasoorya RC \& Gamble GD. Factors influencing mortality in acromegaly. Journal of Clinical Endocrinology and Metabolism 200489 667-674. (doi:10.1210/jc.2003-031199)

27 Kauppinen-Makelin R, Sane T, Reunanen A, Valimaki MJ, Niskanen L, Markkanen H, Loyttyniemi E, Ebeling T, Jaatinen P, Laine $\mathrm{H}$ et al. A nationwide survey of mortality in acromegaly. Journal of Clinical Endocrinology and Metabolism 200590 4081-4086. (doi:10.1210/ jc.2004-1381)

28 Abosch A, Tyrrell JB, Lamborn KR, Hannegan LT, Applebury CB \& Wilson CB. Transsphenoidal microsurgery for growth hormonesecreting pituitary adenomas: initial outcome and long-term results. Journal of Clinical Endocrinology and Metabolism 199883 3411-3418. (doi:10.1210/jcem.83.10.5111)

29 Arita K, Kurisu K, Tominaga A, Eguchi K, Iida K, Uozumi T \& Kasagi F. Mortality in 154 surgically treated patients with acromegaly - a 10-year follow-up survey. Endocrine Journal 200350 163-172. (doi:10.1507/ endocri.50.163)

30 Biermasz NR, Dekker FW, Pereira AM, van Thiel SW, Schutte PJ, van Dulken H, Romijn JA \& Roelfsema F. Determinants of survival in treated acromegaly in a single center: predictive value of serial insulinlike growth factor I measurements. Journal of Clinical Endocrinology and Metabolism 200489 2789-2796. (doi:10.1210/jc.2003-032041)

31 Mestron A, Webb SM, Astorga R, Benito P, Catala M, Gaztambide S, Gomez JM, Halperin I, Lucas-Morante T, Moreno B et al. Epidemiology, clinical characteristics, outcome, morbidity and mortality in acromegaly based on the Spanish Acromegaly Registry (Registro Espanol de Acromegalia, REA). European Journal of Endocrinology 2004151 439-446. (doi:10.1530/eje.0.1510439)

32 Trepp R, Stettler C, Zwahlen M, Seiler R, Diem P \& Christ ER. Treatment outcomes and mortality of 94 patients with acromegaly. Acta Neurochirurgica 2005147 243-251 discussion 250-251. (doi:10.1007/ s00701-004-0466-2)

33 Arosio M, Reimondo G, Malchiodi E, Berchialla P, Borraccino A, De Marinis L, Pivonello R, Grottoli S, Losa M, Cannavo S et al. Predictors of morbidity and mortality in acromegaly: an Italian survey. European Journal of Endocrinology 2012167 189-198. (doi:10.1530/EJE-12-0084)
34 Bex M, Abs R, T'Sjoen G, Mockel J, Velkeniers B, Muermans K \& Maiter D. AcroBel - the Belgian registry on acromegaly: a survey of the 'real-life' outcome in 418 acromegalic subjects. European Journal of Endocrinology 2007157 399-409. (doi:10.1530/ EJE-07-0358)

35 Bogazzi F, Colao A, Rossi G, Lombardi M, Urbani C, Sardella C, Iannelli A, Scattina I, Manetti L, Del Sarto S et al. Comparison of the effects of primary somatostatin analogue therapy and pituitary adenomectomy on survival in patients with acromegaly: a retrospective cohort study. European Journal of Endocrinology 2013169 367-376. (doi:10.1530/EJE-13-0166)

36 Sandret L, Maison P \& Chanson P. Place of cabergoline in acromegaly: a meta-analysis. Journal of Clinical Endocrinology and Metabolism 201196 1327-1335. (doi:10.1210/jc.2010-2443)

37 Sherlock M, Fernandez-Rodriguez E, Alonso AA, Reulen RC, Ayuk J, Clayton RN, Holder G, Sheppard MC, Bates A \& Stewart PM. Medical therapy in patients with acromegaly: predictors of response and comparison of efficacy of dopamine agonists and somatostatin analogues. Journal of Clinical Endocrinology and Metabolism 200994 1255-1263. (doi:10.1210/jc.2008-1420)

38 Bates AS, Van't Hoff W, Jones JM \& Clayton RN. An audit of outcome of treatment in acromegaly. Quarterly Journal of Medicine 199386 293-299.

39 Beauregard C, Truong U, Hardy J \& Serri O. Long-term outcome and mortality after transsphenoidal adenomectomy for acromegaly. Clinical Endocrinology 200358 86-91. (doi:10.1046/j.1365-2265.2003. 01679.x)

40 Tomlinson JW, Holden N, Hills RK, Wheatley K, Clayton RN, Bates AS, Sheppard MC \& Stewart PM. Association between premature mortality and hypopituitarism. West Midlands Prospective Hypopituitary Study Group. Lancet 2001357 425-431. (doi:10.1016/S0140-6736 (00)04006-X)

41 Brada M, Burchell L, Ashley S \& Traish D. The incidence of cerebrovascular accidents in patients with pituitary adenoma. International Journal of Radiation Oncology, Biology, Physics 199945 693-698. (doi:10.1016/S0360-3016(99)00159-5)

Received 14 December 2013

Revised version received 12 May 2014

Accepted 30 May 2014 